\title{
In Vitro Bioactivity and Setting Times of White Portland Cement Combined with Different Radio Pacifying Agents
}

\author{
Nichola Jayne Coleman ${ }^{1, a}$, Rajitha Hanarasinghe ${ }^{2}$, Zeynep Aslı Güçlü ${ }^{3}$ and Samantha Ellen Booth ${ }^{1}$ \\ ${ }^{1}$ University of Greenwich, Faculty of Engineering and Science, Chatham Maritime, Kent, ME4 4TB, UK \\ ${ }^{2}$ Faculty of Medicine, University of Peradeniya, Galaha Road 20400, Peradeniya, Sri Lanka \\ ${ }^{3}$ Department of Paediatric Dentistry, Erciyes University, 38039 Kayseri, Turkey
}

\begin{abstract}
Commercial formulations based on 80:20 mixtures of Portland cement and bismuth oxide (a radiopacifying agent) are used in dentistry as root-filling materials. This study compares the impact of two alternative radiopacifiers, barium sulphate and zirconium oxide, with that of bismuth oxide, on the setting times and bioactivity of white Portland cement. The findings indicate that bismuth oxide prolongs both the initial and final setting times of the cement, and that barium sulphate and zirconium oxide have no effect on this parameter. Hydroxyapatite (HA) formed on the surfaces of all test samples within 7 days of exposure to simulated body fluid, indicating that they possess the potential to stimulate new hard tissue formation. Fourier transform infrared spectroscopy, the traditional technique for the identification of HA, was not appropriate for the analysis of these cement systems owing to the overlap of signals from each of the radiopacifiers with the characteristic P-O bending modes of $\mathrm{HA}$ in the $570-610 \mathrm{~cm}^{-1}$ region. In this respect, the P-O band at $965 \mathrm{~cm}^{-1}$ of HA in the Raman spectrum was found to be a suitable means of detection since it is discrete with respect to all signals arising from the radiopacifying agents and cement phases.
\end{abstract}

\section{Introduction}

Mineral trioxide aggregate (MTA) is a commercial dental cement comprising a mixture of $80 \mathrm{wt} \%$ Portland cement and $20 \mathrm{wt} \%$ bismuth oxide $\left(\mathrm{Bi}_{2} \mathrm{O}_{3}\right)$, a radiopacifying agent, which has been used in endodontic restorations for the past two decades [1]. Bismuth oxide does not directly engage in the hydration reactions of Portland cements; however, its physical presence in the cement system is reported to retard the hydration processes, to increase the porosity of the cement matrix and to decrease mechanical stability [2, 3]. Bismuth oxide is also reported to discolour dental tissues [4].

Barium sulphate $\left(\mathrm{BaSO}_{4}\right)$ and zirconium oxide $\left(\mathrm{ZrO}_{2}\right)$ are among a range of alternative radiopacifying agents with potentially superior chemical, mechanical and biological properties that are currently under investigation for use in hydraulic calcium silicate cements for dental restorations [4-7]. Both barium sulphate and zirconium oxide, by virtue of their low solubilities, are currently used as radiographic contrast agents in a number of biomaterials $[5,8]$.

In this study, experimental cements were prepared with $80 \mathrm{wt} \%$ white Portland cement (WPC) and $20 \mathrm{wt} \%$ of either barium sulphate or zirconium oxide. The in vitro bioactivities of these experimental cements were tested by exposure to simulated body fluid [9], and compared with those of unblended

\footnotetext{
${ }^{\mathrm{a}}$ Corresponding author: n.coleman@gre.ac.uk
} 
WPC and WPC blended with $20 \mathrm{wt} \%$ bismuth oxide (i.e. the proprietary radiopacifier). The impact of the various radiopacifiers on initial and final setting times were also determined using a Vicat apparatus.

\section{Materials and methods}

\subsection{Materials and sample preparation}

The WPC used in this study was supplied by Lafarge [8]. Anhydrous barium sulphate, bismuth oxide, zirconium oxide and hydroxyapatite (HA), ex. Sigma Aldrich, UK, were used without further purification. Cement paste samples were prepared by mixing $10 \mathrm{~g}$ of WPC with $3.5 \mathrm{~g}$ of distilled water. The specimens were sealed into polypropylene tubes and cured at $37{ }^{\circ} \mathrm{C}$ (i.e. body temperature) for 24 hours. Samples blended with barium sulphate, bismuth oxide or zirconium oxide (viz. WPC-Ba, WPC-Bi and WPC-Zr, respectively) were prepared similarly with $20 \mathrm{wt} \%$ replacement of WPC for radiopacifier (at the same water:cement ratio of 0.35 by mass). Each specimen was prepared in duplicate.

\subsection{Initial and final setting times}

Initial and final setting times of the cement pastes were determined by the method described in ASTM C191-08 using a manual Vicat apparatus [8]. Setting data for each cement were collected in triplicate and were subjected to a two-tailed $t$-test at $\mathrm{P}=0.05$.

\subsection{In vitro bioactivity}

Simulated body fluid (SBF) was prepared by the method described in reference $9.0 .15 \mathrm{~g}$ of cement sample were immersed in $150 \mathrm{~cm}^{3}$ of SBF in sealed polypropylene containers at $37^{\circ} \mathrm{C}$ for $3,6,24,48$, 72 and 168 hours. Each analysis was carried out in triplicate. The supernatant solution concentrations of $\mathrm{Si}, \mathrm{Al}, \mathrm{Ca}, \mathrm{P}, \mathrm{Ba}, \mathrm{Bi}$ and $\mathrm{Zr}$ species were determined by inductively coupled plasma analysis (ICP) using a TJA Iris simultaneous ICP-OES. The recovered cement samples were rinsed with deionised water, dried in air at $37^{\circ} \mathrm{C}$ for $24 \mathrm{~h}$ and analysed by Fourier transform infrared spectroscopy (FTIR) using a Perkin Elmer Paragon spectrometer and Raman spectroscopy using a LabRam I Raman instrument fitted with an Olympus BX 40 microscope at $633 \mathrm{~nm}$ wavelength and a spot size of $5 \mu \mathrm{m}$. FTIR and Raman spectra were also collected for synthetic hydroxyapatite, each radiopacifying agent, and the cement samples (WPC, WPC-Ba, WPC-Bi and WPC-Zr) after curing for 7 days at $37^{\circ} \mathrm{C}$.

\section{Results and discussion}

\subsection{Initial and final setting times}

The initial and final setting times for samples WPC, WPC-Ba, WPC-Bi and WPC-Zr were obtained using a manual Vicat apparatus in accordance with ASTM C191-08, and are listed in Table 1.

Table 1. Initial and final setting times for WPC, WPC-Ba, WPC-Bi and WPC-Zr.

\begin{tabular}{|c|c|c|c|c|}
\hline Cement & WPC & WPC-Ba & WPC-Bi & WPC-Zr \\
\hline Initial set (min) & $150 \pm 15$ & $160 \pm 10$ & $255 \pm 15$ & $165 \pm 5$ \\
\hline Final set (min) & $200 \pm 17$ & $197 \pm 6$ & $345 \pm 28$ & $198 \pm 8$ \\
\hline
\end{tabular}

These data demonstrate that the presence of $20 \mathrm{wt} \%$ bismith oxide significantly increases both the initial and final setting times of WPC by $105 \mathrm{~min}$ and $145 \mathrm{~min}$, respectively; whereas, barium sulphate 
and zirconium oxide have no impact on either setting time. These findings confirm those of other studies that also report an increase in setting time associated with bismuth oxide [2,3]. A decrease in setting times has previously been reported for the same white Portland cement containing 20 wt $\%$ zirconium oxide at a water: cement ratio of 0.375 [8]. The apparent discrepancy between these findings may be attributed to the lower water: cement ratio used in this study.

The ability of a radiopacifying agent to increase setting time is a distinct disadvantage, as a common problem regarding MTA is that its setting time (which is reported to vary between 50 min and $2 \mathrm{~h} 45 \mathrm{~min}$ ) is already inconveniently long from a clinical perspective [10]. In this respect, barium sulphate and zirconium oxide are both superior to bismuth oxide, the proprietary radiopacifying agent.

\subsection{In vitro bioactivity}

The formation of a surface layer of substituted HA, $\left(\mathrm{Ca}_{10}\left(\mathrm{PO}_{4}\right)_{6}(\mathrm{OH})_{2}\right)$, on a material placed in SBF solution provides an indication of its bioactivity (i.e. the ability of the material to bond with living bone tissue and to subsequently stimulate tissue regeneration) [9].

The FTIR spectra of HA, barium sulphate, bismuth oxide and zirconium oxide are shown in Fig. 1a. These FTIR spectra demonstrate that various vibrational modes of each of the radiopacifiers overlap with the characteristic P-O bending mode of crystalline HA at $570-610 \mathrm{~cm}^{-1}$, which is commonly used to identify deposits of HA on the surface of materials after exposure to SBF [11].
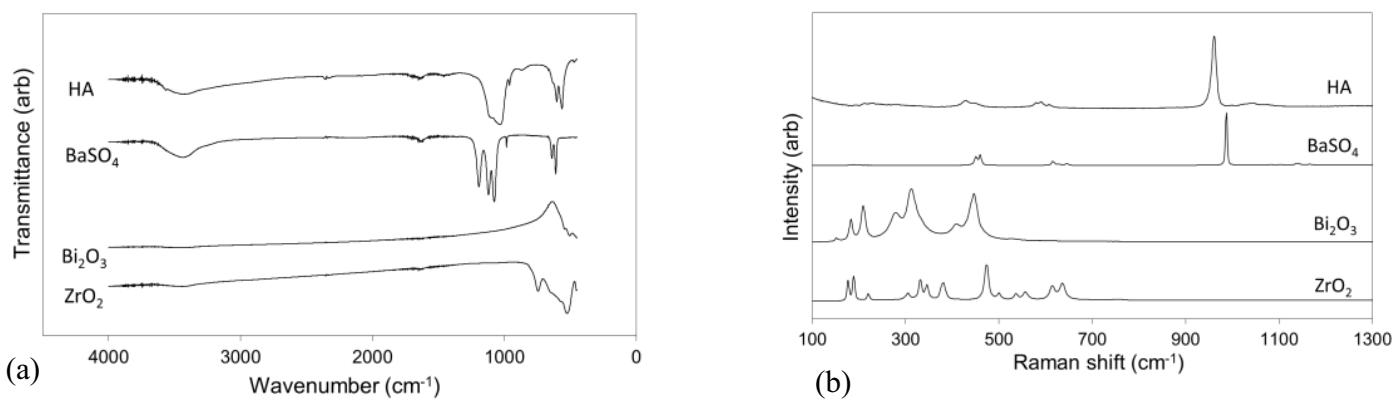

Figure 1. (a) FTIR spectra; and (b) Raman spectra of hydroxyapatite, barium sulphate, bismuth oxide and zirconium oxide.

The Raman spectra of HA, barium sulphate, bismuth oxide and zirconium oxide are shown in Fig. 1 b. The Raman spectrum of each of these mineral phases closely resembles those in the literature [1215]. These spectra demonstrate that the phosphate stretching mode of HA at $965 \mathrm{~cm}^{-1}$ is discrete with respect to all Raman-active bands arising from the radiopacifying agents.

The FTIR and Raman spectra of the experimental cements cured for 7 days are shown in Fig. 2, and those of the cements that were immersed in SBF for 7 days are presented in Fig. 3.
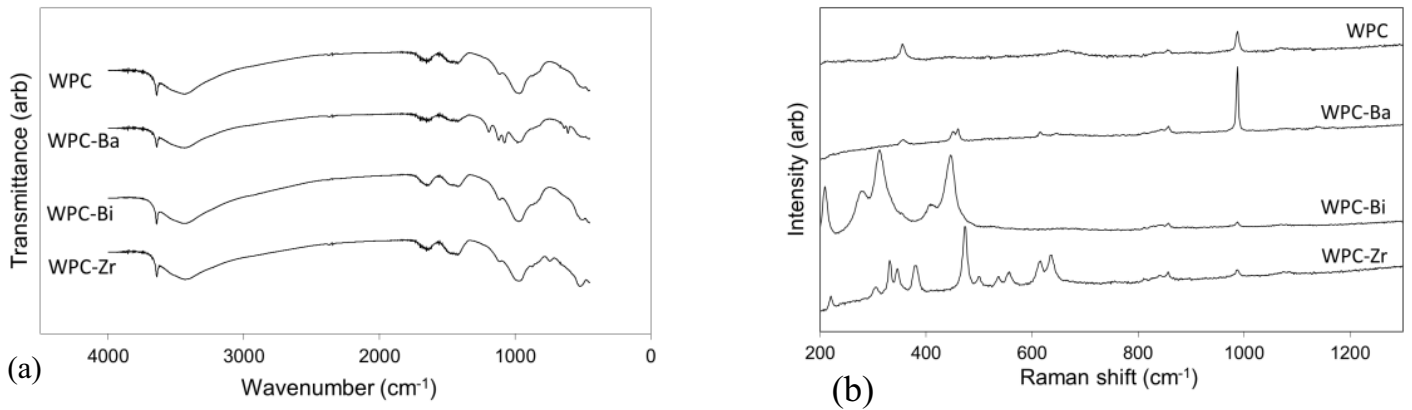

Figure 2. (a) FTIR spectra; and (b) Raman spectra of samples WPC, WPC-Ba, WPC-Bi and WPC-Zr, after curing for 7 days at $37^{\circ} \mathrm{C}$. 
In the FTIR spectra of the cured cements (Fig. 2a), the band at $\sim 970 \mathrm{~cm}^{-1}$ is assigned to Si-O stretching vibrations of the C-S-H gel cement hydration product, with contributions at 870 and 1120 $\mathrm{cm}^{-1}$ from carbonate and sulphate groups, respectively [11]. Carbonate species also give rise to the broad unresolved doublet centred at $1470 \mathrm{~cm}^{-1}$ and the signals at 1660 and $3470 \mathrm{~cm}^{-1}$ are attributed to $\mathrm{O}-\mathrm{H}$ vibrations of water and hydroxyl groups of various hydrated cement phases. The sharp signal at $3670 \mathrm{~cm}^{-1}$ is assigned to the stretching mode of $\mathrm{O}-\mathrm{H}$ in the crystalline calcium hydroxide hydration product. Bands arising from the various vibrational modes of the radiopacifying agents are noted to contribute to the FTIR spectra of the blended cement samples and are particularly apparent in the 550 - $630 \mathrm{~cm}^{-1}$ region.

Raman signals of most hydrated cement phases are inherently weak, and so the spectra of hydrated Portland cements tend to be dominated by bands arising from sulphate bending and stretching modes of unreacted gypsum and ettringite [16]. In the Raman spectra of the cured cements (Fig. 2b), the bands at 986 and $853 \mathrm{~cm}^{-1}$ arise from sulphate stretching modes and the band at $354 \mathrm{~cm}^{-1}$ is assigned to sulphate bending [16]. The signals arising from the radiopacifiers in the Raman spectra of the blended cements are considerably stronger than those of the cement phases (Fig. 2b).

The FTIR spectra of the cement samples following immersion in SBF are shown in Fig. 3a. In the spectrum of WPC, the doublet at 570 and $607 \mathrm{~cm}^{-1}$ is indicative of the formation of crystalline HA which confirms the bioactivity of this material. The dissolution of calcium hydroxide is also demonstrated by the absence of the sharp $\mathrm{O}-\mathrm{H}$ signal at $3670 \mathrm{~cm}^{-1}$ following immersion in SBF. In the FTIR spectra of the blended cements, it is difficult to determine whether HA deposition has occurred, as bands from the radiopacifying agents obscure the region in which the characteristic P-O signal of HA appears (Fig. 3a). Conversely, the characteristic Raman-active phosphate stretching band of HA at $965 \mathrm{~cm}^{-1}$ is clearly and unambiguously observed in the Raman spectra of all samples following immersion in SBF (Fig. 3b).

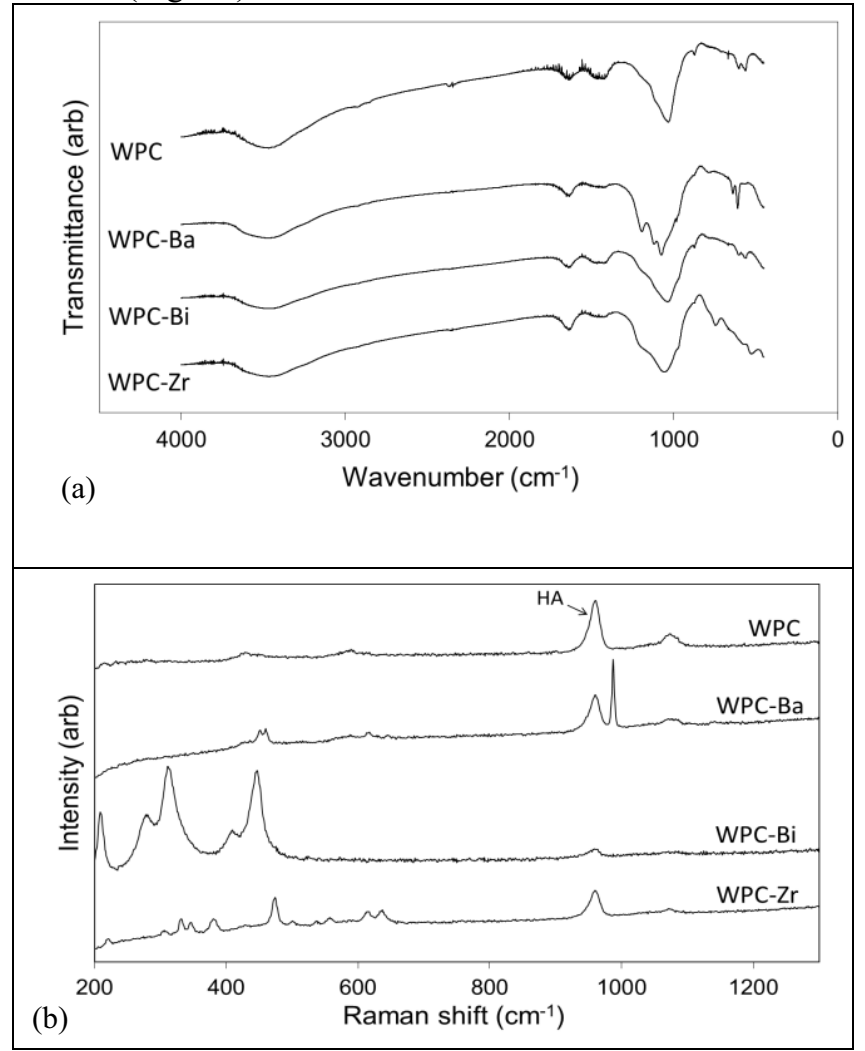

Figure 3. (a) FTIR spectra; and (b) Raman spectra of samples WPC, WPC-Ba, WPC-Bi and WPC-Zr, following immersion in SBF for 7 days at $37^{\circ} \mathrm{C}$. 


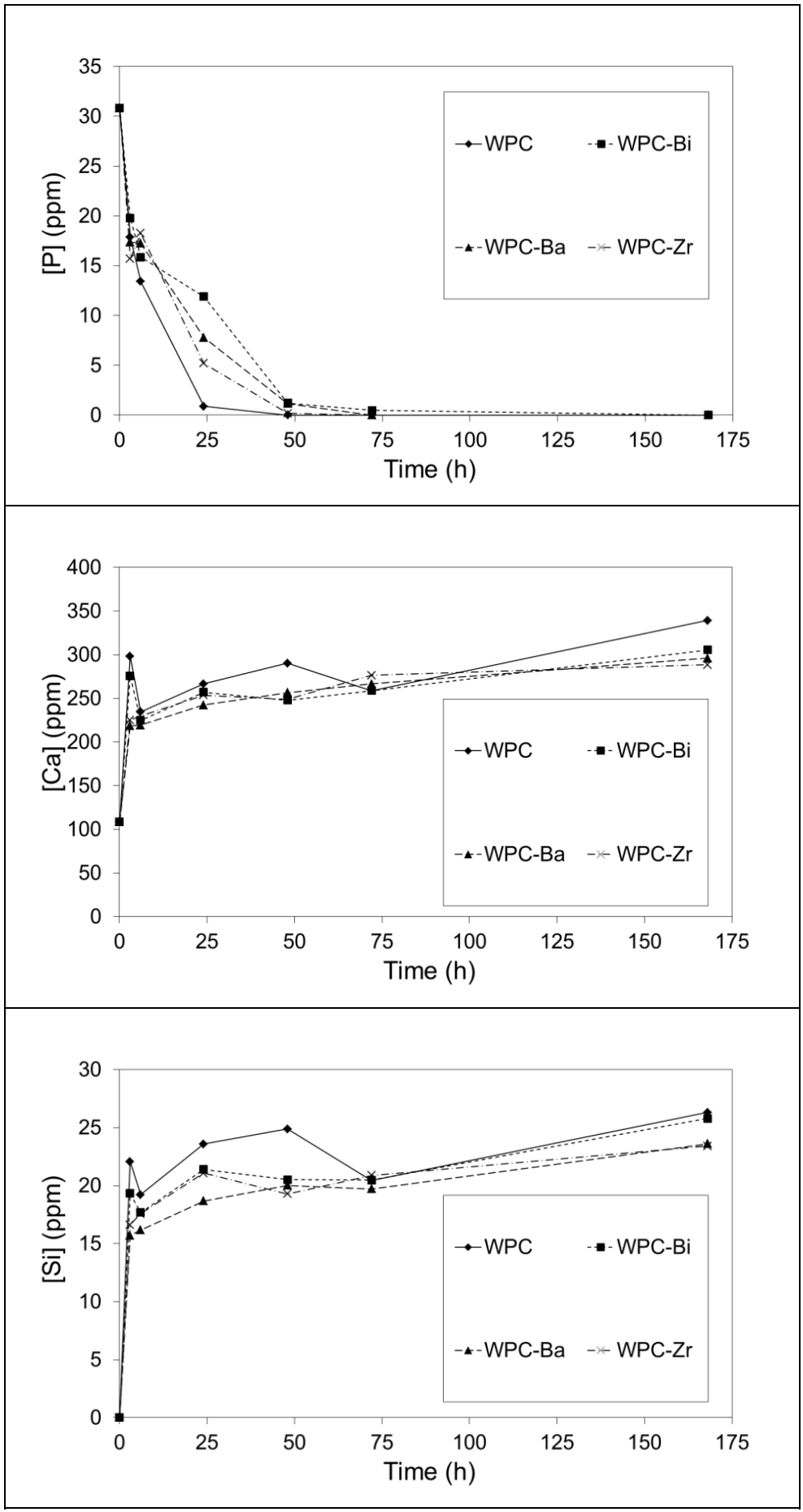

Figure 4. SBF solution profiles for samples WPC, WPC-Ba, WPC-Bi and WPC-Zr.

The concentration profiles of $\mathrm{P}, \mathrm{Ca}$ and $\mathrm{Si}$ in the supernatant SBF solutions in contact with the cement samples are shown in Fig. 4. In all cases, the ongoing reduction of phosphate species in the SBF solution corresponds with the precipitation of HA on the surfaces of the cements and the concurrent release of calcium ions is predominantly attributed to the dissolution of calcium hydroxide 
from the cement matrix. After 24 hours, the extent of uptake of phosphate ions by the cements was found to be of the following order: WPC $>$ WPC-Zr $>$ WPC-Ba $>$ WPC-Bi, indicating that the initial rate of HA deposition was the slowest for the cement mixed with the proprietary radiopacifier. However, after 1 week, the SBF solutions in contact with all cements were essentially depleted of phosphate ions, indicating that there is little difference among the bioactivities of the different cements.

The observed dissolution profiles of $\mathrm{Ca}$ and $\mathrm{Si}$ indicate that the incorporation of the radiopacifiers does not compromise the release of these species from the cement matrix. The release of $\mathrm{Ca}$ and $\mathrm{Si}$ is acknowledged to be relevant to the promotion of new tissue formation, and in this respect all of the experimental radiopacifiers performed similarly [11]. It should be noted, that throughout the duration of the investigation, the concentrations of $\mathrm{Al}, \mathrm{Ba}, \mathrm{Bi}$ and $\mathrm{Zr}$ were found to be below the limit of detection $(\sim 0.1 \mathrm{ppm})$ in $\mathrm{SBF}$, which indicates that they are essentially insoluble in these cement matrices.

Recent studies have indicated that a number of candidate radiopacifiers, including barium sulphate and zirconium oxide, may possess superior physicochemical, mechanical or biological properties to those of bismuth oxide [3-8]. Mineral trioxide aggregate is expensive, and accordingly, Portland cement is currently being considered as an alternative root-filling material which could be mixed with a suitable radiopacifier by the clinician [4, 5-7]. In this respect, the findings of this study indicate that barium sulphate and zirconium oxide are superior to bismuth oxide with respect to setting time. This study has also demonstrated that there is no significant difference in the hydroxyapatite-forming ability or Ca- and Si-release among the experimental cements blended with any of the radiopacifiers.

\section{Conclusions}

This paper reports the comparative impact of $20 \mathrm{wt} \%$ of barium sulphate, zirconium oxide or bismuth oxide on the setting times and in vitro bioactivity of white Portland cement. The proprietary radiopacifying agent, bismuth oxide, prolonged the initial and final setting times of the cement; whereas, the two alternative radiopacifiers were found to have no effect on this parameter. The in vitro bioactivity of all cements was confirmed by the formation of hydroxyapatite on their surfaces in simulated body fluid (SBF) within 7 days, and the release of Ca and Si species from the cement matrix was not compromised by the presence of any of the radiopacifying agents. Fourier transform infrared spectroscopy, the traditional technique for the identification of HA in bioactivity studies, was not suitable for the analysis of these cement systems owing to the overlap of signals from each of the radiopacifiers with the characteristic P-O bending modes of $\mathrm{HA}$ in the $570-610 \mathrm{~cm}^{-1}$ region of the spectrum. In this respect, the $\mathrm{P}-\mathrm{O}$ band at $965 \mathrm{~cm}^{-1}$ in the Raman spectrum was found to be a more appropriate means of detection of HA since it is discrete with respect to all other signals arising from the radiopacifying agents and cement phases.

\section{References}

1. Q. Li, N.J. Coleman, Dent. Mater. J. 34, 458 (2015)

2. B.W. Darvell, R.C.T. Wu, Dent. Mater. 27, 407 (2011)

3. K.S. Coomaraswamy, P.J. Lumley, M.P. Hofmann, J. Endod. 33, 295 (2007)

4. N. Marques, N. Lourenço Neto, A.P. Fernandes, C. Rodini, M. Hungaro Duarte, D. Rios, M.A. Machado, T. Oliviera, J. Microscopy 260, 281 (2015)

5. E.A. Bortoluzzi, J.M. Guerreiro-Tanomaru, M. Tanomaru-Filho, M.A.H. Duarte, Oral Surg. Oral Med. Oral Pathol. Oral Radiol. Endod. 108, 628 (2009)

6. D. Antonijević, D. Ilić, V. Medić, S. Dodić, K. Obradović-Djuričić, Z. Rakočević, Vojnosanitetski Pregled 71, 1006 (2014)

7. M.A.H. Duarte, G.D.D. El Kadre, R.R. Vivan, J.M. Guerreiro-Tanomaru, M. Tanomaru-Filho, I.G. de Moraes, J. Endod. 35, 737 (2009)

8. N.J. Coleman, Q. Li, Mater. Sci. Eng. C, 33, 427, (2013) 
9. T. Kokubo, H. Takadama, Biomaterials 27, 2907 (2006)

10. B. Czarnecka, N.J. Coleman, H. Shaw, J.W. Nicholson, Dent. Med. Probl. 45, 5 (2008)

11. N.J. Coleman, J.W. Nicholson, K. Awosanya, Cement Concr. Res. 37, 1518 (2004)

12. P. Taddei, A. Tinti, M.G. Gandolfi, P.L. Rossi, C. Prati, J. Raman Spec. 40, 1858 (2009)

13. M.G. Gandolfi, K. Van Landuyt, P. Taddei, E. Modena, B. Van Meerbeek, C. Prati, J. Endod. 36, $851(2010)$

14. S.N. White, Chem. Geol. 259, 240 (2009)

15. I. Dobrosz-Gómez, M.A. Gómez-García, J. Bojarska, M. Kozanecji, J.M. Rynkowski, Comptes Rendus Chimie 18, 1094 (2015)

16. N. Garg, K. Wang, S.W. Martin, Cement Concr. Res. 53, 91 (2013) 